\title{
DEPENDENCE OF THE CORRELATION OF SMALL SCALE PHOTOSPHERIC STRUCTURES UPON RESOLUTION
}

\author{
R. G. TESKE and G. H. ELSTE \\ The University of Michigan, Dept. of Astronomy, Ann Arbor, Mich. 48109, U.S.A.
}

(Received 26 July, 1978; in revised form 22 January, 1979)

\begin{abstract}
Correlations between continuum intensity, velocity, and equivalent widths of two $\mathrm{Mn} \mathrm{I}$ lines as observed with two different entrance apertures tend to deteriorate with improvement in spatial resolution. The KPNO multichannel magnetograph was used to make area scans at the center of the disk with entrance apertures $3.5^{\prime \prime} \times 2.5^{\prime \prime}$ arc and $1^{\prime \prime} \times 1^{\prime \prime}$ arc. A coherence analysis shows that this effect is caused by marked differences of fluctuations in temperature and temperature gradients as well as in the velocity structure of photospheric elements of various sizes.
\end{abstract}

\section{Introduction}

A description of the nature of the photospheric inhomogeneities has been sought for in terms of the spatial and temporal distribution of line shifts and those photometric quantities that can be used to specify temperature. According to presently accepted ideas, the inhomogeneities consist of two components: an oscillatory part (Leighton et al., 1962; Deubner, 1975; Rhodes et al., 1977) that is principally a velocity feature, and a part that is associated with the brightness granulation (Reiling, 1971; Canfield and Mehltretter, 1973). Lynch and Chapman (1975) have attempted to separate the two velocity components and to study their statistical properties. There is still a need to understand the temperature structure in the two components.

We have obtained low noise velocity and photometric measurements at two different resolutions on the Sun (Elste and Teske, 1978). In analyzing these data we found a marked tendency for correlations of quantities to be poorer at the higher resolution than at the lower resolution. A coherence analysis has shown that the effect is largely due to marked differences in the instantaneous vertical velocity and temperature profiles in oscillating and non-oscillating cells.

\section{Observations and Reductions}

Two-dimensional raster scans at disk center were made with the magnetograph (Livingston and Harvey, 1971) at the McMath Telescope on Kitt Peak. Simultaneously measured at each raster point were the continuum intensity of $\lambda 5416$, the intensity in two bands completely engulfing each of the lines $\mathrm{Mn} \mathrm{r} \lambda 5395$ and $\lambda 5420$, and also a velocity signal and a magnetic signal in both $\mathrm{Mn} \mathrm{I} \lambda 5395$ and in $\mathrm{Fe}_{\mathrm{I}} \lambda 5393$. The reduction of these data, their calibration and their noise, are described elsewhere (Elste and Teske, 1978). We used magnetograph entrance apertures of $3.5^{\prime \prime} \times 2.5^{\prime \prime}$ arc and $1^{\prime \prime} \times 1^{\prime \prime}$ arc; during the best observations the seeing disk was about $1^{\prime \prime}$ arc or better. No seeing corrections have been introduced. 
Parabolic surfaces were fitted to each datum in each raster scan. Fluctuations in velocity, and relative fluctuations in continuum intensity and equivalent width were measured at each raster point relative to that mean surface. For convenience we introduce the $\delta$-symbol to stand for $\delta I_{c}=\left(I_{c}-\left\langle I_{c}\right\rangle\right) /\left\langle I_{c}\right\rangle$. It is these fluctuations that we have correlated with one another, calculating the correlation coefficients for our three best $1^{\prime \prime} \times 1^{\prime \prime}$ rasters using the standard linear regression formula. The mean values are shown in columns two and three of Table I. In the Table, the quoted 'errors' are the maximum excursion from the mean correlation coefficient for the three rasters. With the exception of the correlation of $V_{\mathrm{Fe}}$ and $\delta I_{c}$ all the correlations in the table deteriorate with improving resolution on the Sun, some markedly, some only slightly. In order to determine whether these results are artifacts of the data-collection process we smoothed data from a $1^{\prime \prime} \times 1$ " raster to yield $4^{\prime \prime} \times 2.4^{\prime \prime}$ arc resolution elements; the results (Table I, column four) resemble those for low resolution data. Finally, we calculated correlations for $1^{\prime \prime} \times 1^{\prime \prime}$ data taken during poor seeing (last column of Table I); these also support the notion that correlations are truly dependent upon the wavenumber that is resolved by the observations.

TABLE I

Correlation coefficients

\begin{tabular}{lllll}
\hline & $\begin{array}{l}3.5^{\prime \prime} \times 2.5^{\prime \prime} \\
\text { aperture }\end{array}$ & $\begin{array}{l}1^{\prime \prime} \times 1^{\prime \prime} \\
\text { aperture }\end{array}$ & $\begin{array}{l}1^{\prime \prime} \times 1^{\prime \prime} \\
\text { smoothed to } \\
4^{\prime \prime} \times 2.4^{\prime \prime}\end{array}$ & $\begin{array}{l}1^{\prime \prime} \times 1^{\prime \prime} \\
\text { in } \\
\text { poor seeing }\end{array}$ \\
\hline$\delta I_{c}$ and $V_{\mathrm{Fe}}$ & $-0.25 \pm 0.03$ & $-0.37 \pm 0.01$ & & -0.32 \\
$\delta I_{c}$ and $\delta W_{5395}$ & $-0.44 \pm 0.02$ & $-0.09 \pm 0.02$ & -0.39 & -0.20 \\
$\delta I_{c}$ and $\delta W_{5420}$ & $-0.48 \pm 0.02$ & $-0.06 \pm 0.02$ & & -0.15 \\
$\delta W_{5395}$ and $\delta W_{5420}$ & $+0.84 \pm 0.00$ & $+0.80 \pm 0.01$ & +0.89 & +0.80 \\
$\delta W_{5395}$ and $V_{\mathrm{Fe}}-0.19 \pm 0.05$ & $-0.16 \pm 0.01$ & & -0.14 \\
$V_{\mathrm{Fe}}$ and $V_{\mathrm{Mn}}$ & $+0.94 \pm 0.01$ & $+0.82 \pm 0.02$ & & +0.81 \\
\hline
\end{tabular}

\section{Coherence and Phase}

We have used the tools of coherence and phase spectra to identify reasons why the observed correlations change with entrance aperture. Edmonds (1962) and Edmonds and Webb (1972a, b) have thoroughly described the calculation and interpretation of coherence and phase spectra. We have followed their prescriptions for smoothing the power spectra, co-spectra and quadrature spectra in calculating phase and coherence. One dimensional spectra were obtained by averaging power, phase and coherence for the 63 individual scan lines of each of the three rasters that were observed with the $1^{\prime \prime} \times 1^{\prime \prime}$ entrance aperture. Mean spatial power spectra that were calculated are smooth, without peaks, and look very much like those reported by many authors (e.g. Edmonds, et al., 1965; Mehltretter, 1971; Lynch and Chapman, 1975). 


\subsection{Correlation of $\delta I_{c}$ AND $V_{\mathrm{Fe}}$}

We use the convention that rising material has negative velocity.

The mean coherence spectrum for three rasters is shown in Figure 1. Observations made with the $3.5^{\prime \prime} \times 2.5^{\prime \prime}$ arc entrance aperture probed the part of the spectrum at $k \lesssim 1.2(\mathrm{Mm})^{-1}$ while the $1^{\prime \prime} \times 1^{\prime \prime}$ entrance aperture examines all fluctuations at

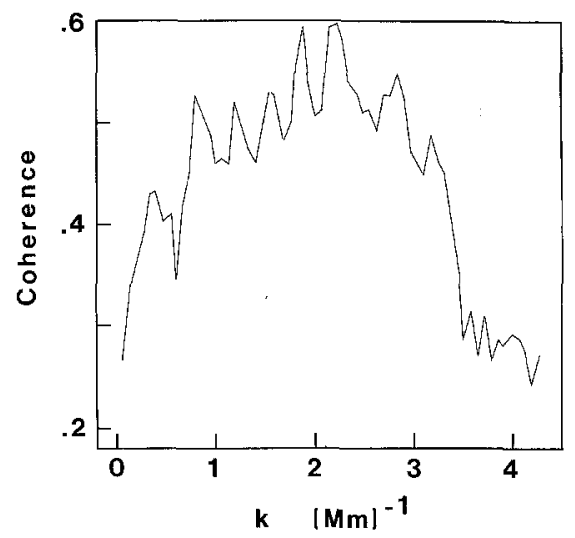

Fig. 1. The mean coherence spectrum of continuum intensity fluctuations and Doppler velocity measured in Fe I $\lambda 5393$.

$k \$ 4.3(\mathrm{Mm})^{-1}$. Because coherence is greatest in the wavenumber range $0.8<k<$ $3.4(\mathrm{Mm})^{-1}$, the observed correlation should improve as we pass from low resolution to the higher resolution, as is in fact found (Table I). We conclude that the observed trend of correlation is due to the relatively modest coherence at large cell sizes and the improved coherence at intermediate cell sizes. Canfield and Mehltretter (1973) calculated mean coherences, as corrected for resolution, in cells of dimension $1.24^{\prime \prime}-2.64^{\prime \prime}$ and $0.80^{\prime \prime}-1.24^{\prime \prime}$, finding, as we do, a small increase in coherence towards the smaller cell sizes. Our mean coherence spectrum is in fair agreement with those of Edmonds et al. (1965; hereafter referred to as EMS). All show an abrupt drop of coherence at $k \sim 3.4(\mathrm{Mm})^{-1}$, where the spectral power of both fluctuations is very low. Significant coherences can exist in wavenumber regions of low spectral power so long as the correlated portions of power dominate the uncorrelated parts. Near the resolution limit in our data, noise fluctuations begin to add important contributions to uncorrelated power and thus erode coherences. EMS first suggested that this effect might account for the observed decline of coherence towards high wavenumbers. Mehltretter (1971) has attempted to correct for the influence of noise; the method requires an accurate knowledge of the noise power spectrum. We do not think our coherences are reliable at wavenumbers $k \geq$ $3.0(\mathrm{Mm})^{-1}$

RMS noise levels in our data were 0.0008 in $\delta I_{c}$ and $11 \mathrm{~m}^{-1} \mathrm{~s}$ in $V_{\mathrm{Fe}}$, very good by ordinary standards. If one hopes to examine the question of coherence of $V$ and $\delta I_{c}$ 
at large wavenumber, these noise levels will have to be considerably improved along with any improvement in resolution.

\subsection{Correlation of $\delta I_{c}$ AND $\delta W_{\lambda}$}

The equivalent widths of the $\mathrm{Mn} \mathrm{I}$ lines are sensitive only to temperature and not to turbulence (Elste and Teske, 1978). For these Fraunhofer lines, $W_{\lambda}$ responds inversely to $T$; thus if temperature perturbations are common to both the lineforming and continuum-forming layers we expect $\delta W_{\lambda}$ and $\delta I_{c}$ to be negatively correlated. Table I shows that they are fairly well negatively correlated at lower resolution but are essentially uncorrelated at the higher resolution.

Mean coherence and phase spectra for three rasters scanned with the $1^{\prime \prime} \times 1^{\prime \prime}$ aperture are shown in Figure 2. Coherence drops to a well pronounced minimum value of 0.16 (mostly uncorrelated power) near $k=1.3(\mathrm{Mm})^{-1}$ and increases then steadily towards higher wavenumbers. Spectral phase is $\sim \pi$ (the expected negative correlation) up to $k \sim 1.0(\mathrm{Mm})^{-1}$ and then rises to nearly zero radians at the highest k. This behavior is found for both the Mn r lines we observed. It is the change in spectral phase that drives the strong negative correlation coefficient of poorly resolved data towards algebraically larger values in the better resolved observations.

From photographic observations of slit spectra EMS calculated a coherence spectrum for $\delta W_{\lambda}$ in the $C_{I} \lambda 5052$ line and $\delta I_{c}$. They found an almost steady decline in the coherence with $\mathbf{k}$ for $k>2.0(\mathrm{Mm})^{-1}$ from 0.9 to 0.5 at $4.0(\mathrm{Mm})^{-1}$. This is unlike the present work on Mn lines, where we find an increase. EMS speculated upon various solar and non-solar causes but reached no conclusion. We believe that the different behavior of the correlations for the carbon and manganese lines with continuum is largely solar, at least in the wavenumber range $k<3.0(\mathrm{Mm})^{-1}$, and that it reflects differences in the instantaneous vertical temperature gradient in structures of large and small scale.

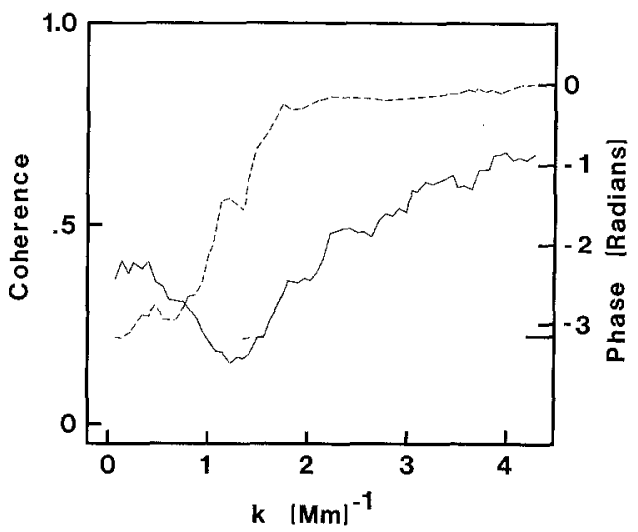

Fig. 2. Mean coherence spectrum (__ ) and phase spectrum (-- - - ) of fluctuations of continuum intensity and equivalent width in the $\mathrm{Mn} \mathrm{I} \lambda 5395$ line. 
Below we make a comparison of EMS results for the carbon line and our results for the manganese lines to explain why we think the effect is solar.

Let us first consider the influence of the noise. According to the dêfinition of equivalent width for weak and medium-strong lines, fluctuations $\mathrm{d} W_{\lambda}$ depend upon line $(L)$ and continuum $(C)$ signals as

$$
\mathrm{d} W_{\lambda}=L C^{-2} \mathrm{~d} C-C^{-1} \mathrm{~d} L
$$

At the highest wavenumbers $\left(k>3.0(\mathrm{Mm})^{-1}\right)$ the spectral power in the line and continuum signals is so very low that noise will dominate signal power there. According to Equation (1), uncorrelated noise fluctuations of $C$ and $L$ will tend to produce a positive correlation of $\delta W_{\lambda}$ and $\delta I_{c}$ for any line. In the case of the neutral carbon line $\lambda 5052$, as analysed by EMS, noise will produce a seeming correlation of the temperature in the line-forming strata with those in the continuum-forming strata. However for our neutral manganese lines the noise will produce the seemingly opposite correlation of temperatures in line- and continuum-forming strata, i.e. hot in one with cold in the other stratum. If equal amounts of noise dominate both the $C$ and $L$ channels at the highest wavenumbers, then we expect to find about as much correlated as uncorrelated power (coherence $\sim 0.5$ ), and the parts of $\delta W_{\lambda}$ and $\delta I_{c}$ ought to appear in phase. This is true for the results of EMS. In our case, the noise $\mathrm{d} C / C=0.00079$ and $\mathrm{d} L / L=0.00030$; we therefore expect the noise-dominated coherence to reach $\sim 0.7$ at the high wavenumber part of the spectrum, as is observed.

A careful examination of the power, coherence and phase spectra shows that the coherence minimum and the transition to zero phase (Figure 2) occur at wavenumbers where significant amounts of fluctuation power still exist and where the above noise hypothesis is certainly not valid. There the observed relations provide a reliable view of the vertical temperature profile of photospheric elements of various sizes.

At wavenumbers $k \leqslant 1.2(\mathrm{Mm})^{-1}$ below the coherence minimum we are observing fluctuations in temperature averaged over larger groups of granules of dimension $>$ $4^{\prime \prime}$ arc, which in the velocity picture may correspond to the time periodic oscillating elements (Rhodes et al., 1977). For the correlated parts of the $\delta W_{\lambda}$ and $\delta I_{c}$ fluctuations the spectral phase in the Mn data is $180^{\circ}$ in these elements, that is, the temperature excursions are in the same sense in the line-forming and in the continuum-forming layers, roughly preserving the mean photospheric temperature gradient. The zero spectral phase between $\delta W_{\lambda}$ and $\delta I_{c}$ found by EMS for the carbon line also supports this view.

However at wavenumbers above the coherence minimum and before the noise starts dominating we are detecting non-oscillating, transient photospheric elements of size $<3^{\prime \prime}$ arc, in which the correlated parts of the $\delta W_{\lambda}$ and $\delta I_{c}$ fluctuations are in phase for both the carbon and manganese data. If we try to interpret this in terms of fluctuations in effective temperature of structures with fixed temperature gradients with optical depth we are faced with a contradiction. While the carbon line requires 
temperatures in line- and continuum-forming strata to vary in phase, the manganese lines indicate the opposite behavior. How can this be understood?

We must realize that for fixed numbers of absorbers steeper source function gradients produce larger line depths in any line. For evaluating the influence of the gradient of the source function on the equivalent widths of weak lines, which vary proportional to the central line depths, we may approximate the integrals over the photospheric layers by mean values of the integrals. With the restriction to disk center and to a linear Planck function in the layers of line formation,

$$
B(\tau)=B(1+\beta \tau)
$$

we find

$$
W_{\lambda} \sim r \sim \frac{\eta}{1+\eta} \frac{\beta}{1+\beta} e^{-\bar{\tau}}
$$

the same functional dependence of $\boldsymbol{\eta}$ and $\boldsymbol{\beta}$, where $\boldsymbol{\eta}$ is the ratio of the line to continuums absorption, and $e^{-\bar{\tau}}$ is the attenuation factor for the depth of line formation. For $\mathrm{Mn} 5395, \eta \sim 10^{+6.7 \theta}$, decreasing with increasing temperature, and for C 5052, $\eta \sim 10^{-8.5 \theta} P_{e} \theta^{-5 / 2}$, increasing with temperature and electron pressure. However, for both lines $\mathrm{d} W_{\lambda} / \mathrm{d} \beta>0$.

To clarify the inferences we have made from the observations, we present in Table II the qualitative behavior of the two line types relative to the continuum, listing from left to right the observed correlations and their inferred interpretation for (i) temperature fluctuations with fixed gradient and for (ii) temperature gradient fluctuations in the strata of line formation. Case $\mathrm{A}$ is the situation for small wavenumbers; Case B is for large wavenumbers and noise. In each case we list the Mn line above the carbon line according to their depth of formation and call attention to the fact that the carbon line is formed rather close to the layer of continuum formation. In Case $\mathrm{A}$, the observed correlation of $\delta W_{\lambda}(\mathrm{Mn})$ and $\delta I_{c}$ requires that the

TABLE II

\begin{tabular}{|c|c|c|c|c|c|}
\hline & & \multirow{3}{*}{$\begin{array}{l}\text { Observed correlation } \\
\text { of equivalent widths } \\
\text { with continuum }\end{array}$} & \multicolumn{2}{|c|}{ Inferred correlations } & \\
\hline & & & $\begin{array}{l}\text { of temperature } \\
\text { fluctuations }\end{array}$ & $\begin{array}{l}\text { of temp. gradient } \\
\text { fluctuations }\end{array}$ & \\
\hline & & & $\begin{array}{l}\text { with temperatur } \\
\text { forming layer }\end{array}$ & n the continuum & \\
\hline \multirow[t]{2}{*}{$\stackrel{\downarrow}{\text { depth }}$} & $W_{\lambda}(\mathrm{Mn})$ & out of phase & in phase & out of phase & Case $\mathrm{A}$ \\
\hline & $W_{\lambda}(\mathrm{C})$ & in phase & in phase & in phase & \\
\hline \multirow[t]{2}{*}{$\underset{\downarrow}{\downarrow}$} & $W_{\lambda}(\mathrm{Mn})$ & in phase & out of phase & in phase & Case B \\
\hline & $W_{\lambda}(\mathrm{C})$ & in phase & in phase & in phase & \\
\hline
\end{tabular}

Interpretation of observed correlations 
temperature gradients steepen with decreasing temperature at the continuum forming level, while the observed correlation of $\delta W_{\lambda}(\mathrm{C} I)$ and $\delta I_{c}$ yields a contradictory prediction. Both of the observed correlations are explained by in-phase temperature fluctuations at all three atmospheric levels and we therefore judge this to be the correct inference for Case A.

Case B holds not only for noise but also for the small scale inhomogeneities. If we interpret the observed correlations as being caused only by temperature fluctuations at the three atmospheric levels, we get contradictory results. The behavior of both lines is however explained by a steepening temperature gradient in the line-forming regions with increasing continuum-level temperature, and vice versa. It is the similarity with the behavior of noise which makes the detection of the stratification in small scale structures difficult. But the existence of sufficient power in this wavenumber regime of the spectra forces us to conclude that in the small scale structures, temperature gradients are correlated with temperatures in the continuum-forming layer at $5000 \AA$. This may indeed lead to an inverse horizontal temperature structure at a higher photospheric level, i.e. dark features separated by narrow bright rims. This is shown clearly in plage regions in the original of a CN $\lambda 3883$ spectroheliogram published by Gillespie (1971) and in $\mathrm{Mg} \mathrm{b}$ spectroheliograms $0.4 \AA$ away from line center published by Beckers (1976).

Let us summarise our findings concerning the two correlation analyses, ours and that by EMS.

Besides the possibility for instrumental effects in the two entirely different observational approaches we may explain the different behavior of the coherences of the manganese lines as compared with that of the carbon line with continuum intensity as follows: For the carbon line with its depth of formation close to that of the continuum the coherence must be expected to stay high with increasing wavenumber and only drop for very small cell sizes as a result of noise. When fine structures are encountered involving regions with different gradients, both higher temperatures and steeper gradients produce the same effect, but not so for the manganese lines. For these lines, which are formed at much higher altitudes, the size of the structures over which the aperture averages becomes important. Stronger gradient variations will be detected more frequently in granulation sized structures than in averages over a larger number of them. And some of the structures of smallest size present in deep layers are going to be smoothed out by horizontal radiative exchange when they reach the manganese-line-forming layers. If the average is extended over one or more complete granules the fine structures will be lost, and due to the different horizontal extent, coherences with continuum structures must drop. Only at very large cell sizes will we detect again a temperature structure approximating the average photospheric temperature profile.

\subsection{Correlation OF $\delta W_{\lambda}$ AND $V_{\mathrm{Fe}}$}

The mean photospheric depths to which our measures of $\delta W_{\lambda}(\operatorname{Mn~I} \lambda 5395)$ and $V_{\mathrm{Fe}}(\mathrm{Fe}$ I $\lambda 5393)$ refer are within $60 \mathrm{~km}$ of one another, with the $V_{\mathrm{Fe}}$ referring to the 
higher layer. Figure 3 shows that there is uniformly low level of coherence between these measures at all wavenumbers $k<3.0(\mathrm{Mm})^{-1}$. Spectral phase for the correlated portions of power is $\pm \pi$ at all $\mathbf{k}$, requiring that rising elements have lower than average temperature and/or steeper temperature gradients. These coherence and phase spectra mean that the correlation coefficients should be negative and closely the same at both resolutions (Table I). It would be hard to envision that the temperature in rising gasses would drop below the average. But if the heat supply for the upper part of the hot columns is predominantly radiative from below, as proposed by Musman and Nelson (1976), it may indeed be the case. Increased continuous absorption in the hot underlying layers would decrease the energy supply and thus would make an inversion of the temperature structure indeed possible.

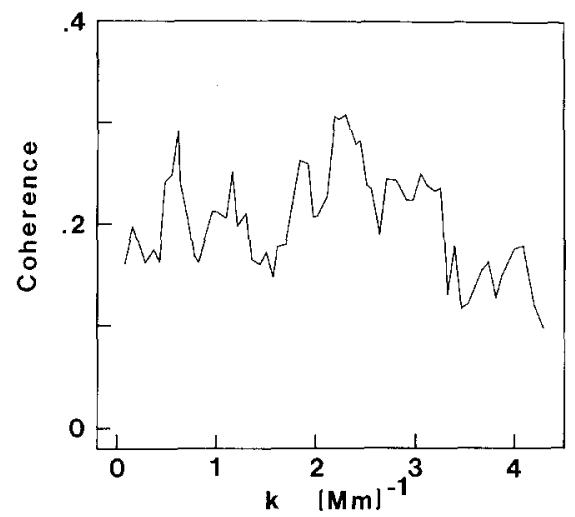

Fig. 3. Mean coherence spectrum of $\delta W_{\lambda}(\mathrm{Mn}$ I $\lambda$ 5395) and Doppler velocity in Fe I $\lambda 5393$.

\subsection{Correlation of $V_{\mathrm{Fe}}$ and $V_{\mathrm{Mn}}$}

The spatial coherence of velocities measured by us simultaneously in Fe $\mathrm{r} \lambda 5393$ and in Mn I $\lambda 5395$ sharply diminishes with increasing wave-number (Figure 4); it is for this reason that the correlation coefficient (Table I) declines as resolution improves. The decline of coherence was already found by EMS who considered the possible effects of spatial resolution in causing reduced coherences, and by Canfield and Mehltretter (1973).

We display in Figure 4 a coherence spectrum of velocities obtained from data taken during a time when the seeing disk was estimated at $2^{\prime \prime}-4$ " arc (see also Table I). The comparison of that spectrum with the good-seeing coherence spectrum indicates that resolution on the Sun plays an important role in limiting coherences at moderate and high wavenumbers; that the spectra agree at $k>3.0(\mathrm{Mm})^{-1}$ is a certain indication that non-coherent noise dominates there, even during good seeing. However, this comparison also gives us confidence that the decline of coherence in the good-seeing spectrum is solar in origin, at least out to $k \sim 2.0(\mathrm{Mm})^{-1}$. 


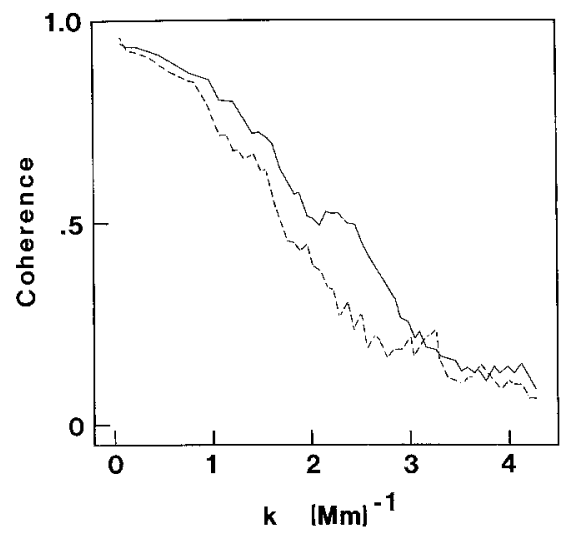

Fig. 4. Mean coherence spectrum of Doppler velocities measured in Fe I $\lambda 5393$ and in Mn I $\lambda 5395$, calculated from three raster frames taken during excellent seeing $(\longrightarrow)$ and taken from one poorseeing raster $(----)$.

In the domain of time-periodic oscillations the coherence declines only slowly with increasing wavenumber. We expect it to be approximately constant if the oscillations are non-propagating evanescent waves (Rhodes et al., 1977) in which velocity is nearly in temporal phase at all depths (Evans et al., 1963; Edmonds et al., 1965; Sivaraman, 1973). In the domain of non-oscillating cells above $k \geq 1.2(\mathrm{Mm})^{-1}$ the coherence declines more steeply with wavenumber, although spectral phase remains zero. At $k \sim 2.0(\mathrm{Mm})^{-1}$ only half the power is correlated, even though the spectrum lines are formed in closely adjacent layers. Thus, motions in the small cells may be chaotic and noise-like. Canfield and Mehltretter (1973) pointed out that the decline of coherence is consistent with the view that small cells are dissolved over a shorter height range than are the larger cells.

\section{Discussion}

As a way of showing that the correlations and coherences calculated here have a definite meaning for the vertical atmospheric structure of the inhomogeneities, we first focus our attention on the wavenumber region of time-periodic oscillations, $k \leq 1.2(\mathrm{Mm})^{-1}$. These elements contain essentially all the fluctuation power in velocity. We have assumed that in these large cells the bulk of the fluctuation power in temperature - and thus in line equivalent width or continuum brightness - is also time-periodic and associated with the oscillations. Under this assumption, it is appropriate to examine the coherences in terms of a model in which fluctuations at a place on the photosphere have a strictly sinusoidal time dependence. In these cells, the velocity-velocity coherence is high and nearly constant with wavenumber. This is consistent with the view that gas motions in the oscillations are nearly in temporal phase at different atmospheric levels, as many others have found (references cited above). Next, the calculated mean coherence of $\delta W_{\lambda}(\mathrm{Mn}$ I $\lambda 5395)$ and $V_{\mathrm{Fe}}$ (about 
0.2 ) in the oscillations means that a positive temperature excursion in the line forming layer ( $\Delta T_{\text {Line }}$ ) is ahead of upward $V_{\mathrm{Fe}}$ by about $100^{\circ}$, consistent with the temporal phase leads of from $90^{\circ}$ to $120^{\circ}$ found by others (see, e.g., Holweger and Testerman, 1975; and references cited by them). Third, the mean coherence of $\delta I_{c}$ and $V_{\mathrm{Fe}}$ for the oscillations requires that a positive $\Delta T_{\text {continuum }}$ is ahead of upward $V_{\mathrm{Fe}}$ by about $60^{\circ}$. A phase lead of $50^{\circ}$ was found by Evans et al. (1962) for intermediate strength Fraunhofer lines, and of about $18^{\circ}$ by Edmonds and Webb (1972b) for velocity in the low-lying C I $\lambda 5052$ line. Last, the coherence of $\delta W_{\lambda}$ and $\delta I_{c}$ in the oscillations (about 0.3 ) means that $\Delta T_{\text {Line }}$ leads $\Delta T_{\text {continum }}$ by about $60^{\circ}-70^{\circ}$, a value that is reasonably consistent with the other temporal phases deduced above and which agrees with Sivaraman's (1973) result $\left(57^{\circ}\right)$. In summary, our coherences for the time-periodic oscillations provide a view of them that is in harmony with results derived by others.

Although it is our aim next to use the coherences for the small, non-oscillating cells which are associated with the brightness granulation to specify their structure, we cannot interpret the coherences in the same manner since no time-periodicities are involved. Nevertheless the coherences already show that the small structures are similar to the oscillations in one way and different in three other ways.

(1) The two kinds of cells are alike in that both show only a weak (negative) correlation of $\Delta T_{\text {Line }}$ and upward $V_{\mathrm{Fe}}$. Both kinds of cells made contributions to the cross-correlations calculated by Canfield and Mehltretter (1973) which also show a tendency for cool material in the line-forming layers to rise.

(2) Gas velocities at different depths in the small cells tend to be non-coherent with one another, unlike the situation in the oscillations. This may mean that the small elements have only a short velocity dissipation length. It may also indicate that the elements have the nature of running waves.

(3) There is a slightly better (positive) correlation of $\Delta T_{\text {continuum }}$ and upward $V_{\mathrm{Fe}}$ in the small cells than in the oscillations, a result which seems in direct disagreement with our interpretation of the low velocity-velocity coherence.

(4) $\Delta T_{\text {Line }}$ and $\Delta T_{\text {continum }}$ are only partly correlated in the small cells. Where correlated temperature fluctuations are found, the instantaneous vertical temperature profile varies substantially from the mean photospheric one, being either steeper or shallower. In contrast, the temperature profile of the oscillating elements seems to remain roughly like the mean photospheric slope.

\section{References}

Beckers, J. M.: 1976, in D. J. Williams (ed.), Proc. Int. Symp. Solar-Terrestrial Physics, American Geophysical Union, p. 89.

Canfield, R. C. and Mehltretter, J. P.: 1973, Solar Phys. 33, 33.

Deubner, F.-L.: 1975, Astron. Astrophys. 44, 371.

Edmonds, F. N. Jr.: 1962, Astrophys. J. 136, 507.

Edmonds, F. N. Jr. and Webb, C. J.: 1972a, Solar Phys. 22, 276.

Edmonds, F. N. Jr. and Webb, C. J.: 1972b, Solar Phys. 25, 44. 
Edmonds, F. N. Jr, Michard, R., and Servajean, R.: 1965, Ann. Astrophys. 28, 534.

Elste, G. H. and Teske, R. G.: 1978, Solar Phys. 59, 275.

Evans, J. W., Main, P., Michard, R., and Servajean, R.: 1962, Astrophys. J. 136, 682.

Evans J. W., Michard, R., and Servajean, R.: 1963, Ann. Astrophys. 26, 368.

Gillespie, B.: 1971, Solar Phys. 21, 93.

Holweger, H. and Testerman, L.: 1975, Solar Phys. 43, 271.

Leighton, R. B., Noyes, R. W., and Simon, G. W.: 1962, Astrophys. J. 135, 474.

Livingston, W. C. and Harvey, J.: 1971, Kitt Peak Nat. Obs. Contr. No. 558.

Lynch, D. K. and Chapman, G. A.: 1975, Astrophys. J. 197, 241.

Mehltretter, J. P.: 1971, Solar Phys. 16, 253.

Musman, S. and Nelson, G. D.: 1976, Astrophys. J. 207, 981.

Reiling, H.: 1971, Solar Phys. 19, 297.

Rhodes, E. J. Jr., Ulrich, R. K., and Simon, G. W.: 1977, UCLA Astronomy and Astrophysics Preprint No. 12.

Sivaraman, K. R.: 1973, Solar Phys. 33, 319. 